\title{
The Role of the Nature of Magnetic Coupling on the Martensitic Transformation in Fe-Ni
}

\author{
M. Acet, E.F. Wassermann, K. Andersen*, A. Murani* and O. Schärpff* \\ Tieftemperaturphysik, Gerhard-Mercator Universität Duisburg, 47048 Duisburg, Germany \\ * Institut Laue-Langevin, BP. 156, 38042 Grenoble cedex 9, France
}

\begin{abstract}
Fe}_{100-\mathrm{x}} \mathrm{Ni}_{\mathrm{x}}$ with $\mathrm{x} \leq 30$ at.\%, known as anti-Invar alloys, undergo martensitic transformations. Total energy calculations have shown that these alloys incorporate a strongly fluctuating magnetic moment in the fcc state. In order to understand the relationship between the moment fluctuations and the martensitic transformation, we have investigated the magnetic moment in the paramagnetic foc state of these alloys by polarized neutron diffraction. We find that the magnetic correlations are ferromagnetic in nature although, the ground state of these alloys are expected to be antiferromagnetic. We further observe that the magnetic moment of the fcc state at $\mathrm{M}$, increases with increasing Ni concentration, indicating that the fcc state is stabilized by increasing ferromagnetic exchange. The results are found to be in agreement with thermodynamical predictions.
\end{abstract}

\section{INTRODUCTION}

The unstable magnetic moment of fcc Fe with respect to a volume change is the origin of the richness of the magnetic features exhibited by fcc-Fe incorporating alloys of the $3 d$ elements $[1,2]$. In the ground state fcc-Fe is in a low-momnet (LM) antiferromagnetic (AF) state with a Néel temperature $T_{N}=65 \mathrm{~K}$, a magnetic moment $\mu=0.5 \mu_{\mathrm{B}}$ and an equilibrium lattice constant of about $\mathrm{a}=3.56 \AA$ (atomic volume $\mathrm{V}_{\mathrm{a}} \approx 11.3$ $\mathrm{nm}^{3}$ ). When the lattice is expanded to above $\mathrm{a}=3.65 \AA\left(\mathrm{V}_{\mathrm{a}} \approx 12.1 \mathrm{~nm}^{3}\right)$ the magnetic coupling in fcc-Fe becomes ferromagnetic (FM) with $\mu=2.8 \mu_{\mathrm{B}}$ and is designated as a high-moment state (HM) [3]. This state lies energetically higher than the LM state by several mRy. The occurrence of these states in $\mathrm{fcc}$ Fe leads to an enhanced anharmonicity of the lattice which manifests itself as an excess thermal expansion, which can be observed at temperatures within the stability range ( 1184 $\mathrm{K}<\mathrm{T}<1665 \mathrm{~K}$ ) [4]. This effect, known as antiInvar, is found in Fe or Mn-rich fcc alloys and is the opposite of the Invar effect [5,6]. In Invar an energetically high lying LM state exists at a volume smaller than that of the equilibrium HM state volume, causing the interatomic potential to be skewed towards smaller volumes (negative anharmonicity). This acts to reduce the normal lattice thermal expansion with increasing temperature up to around the Curie temperature $\mathbf{T}_{\mathbf{c}}$. Both in Invar and anti-Invar the magnetic moment changes rapidly with volume leading to strong longitudinal moment fluctuations at finite

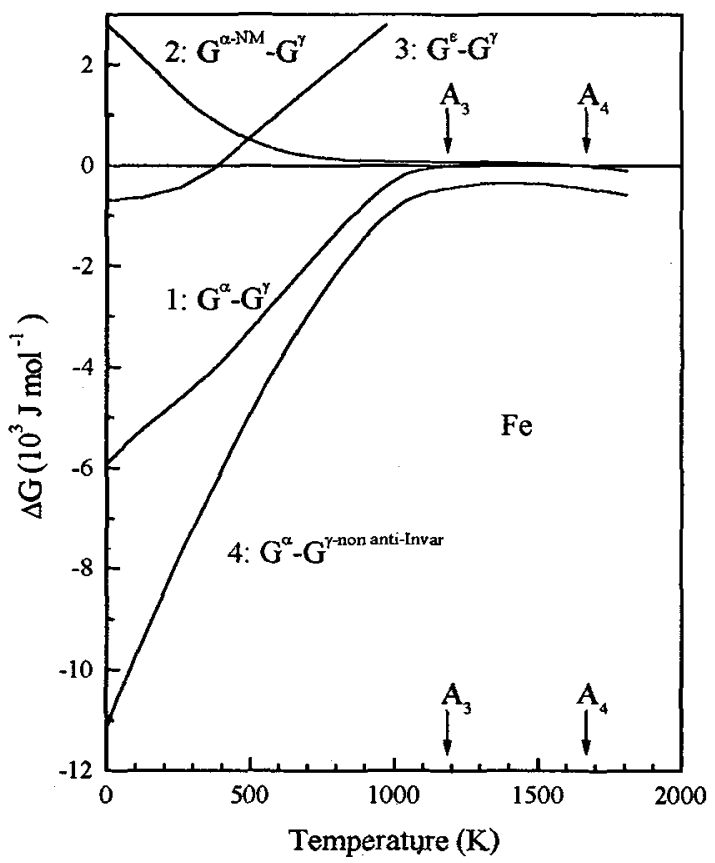

Fig. 1. The differences in the Gibbs free energy of bcc, fcc and hcp Fe. Without anti-Invar fcc Fe is not stable with respect to bcc Fe. 


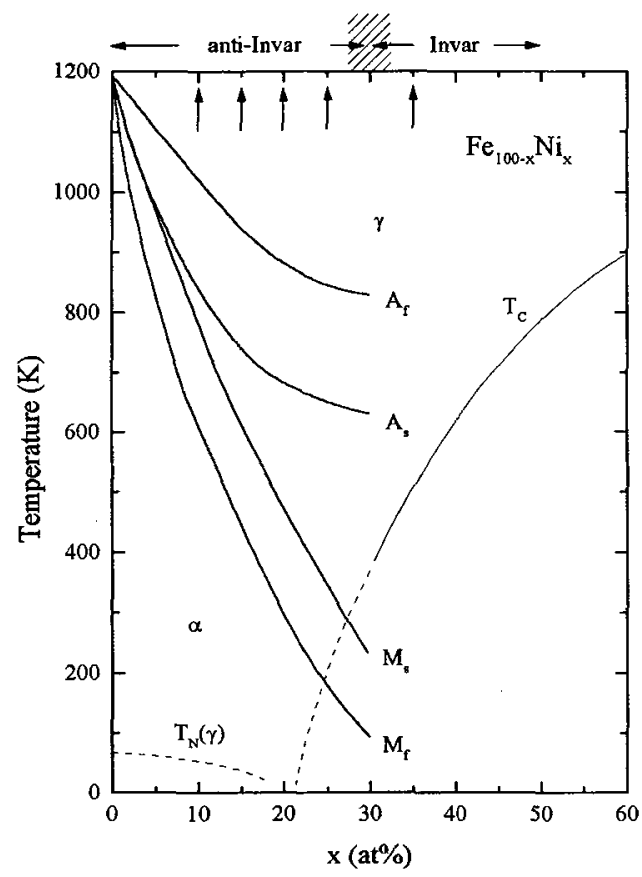

Fig. 2. The phase diagram of $\mathrm{Fe}_{100-\mathrm{x}} \mathrm{Ni}_{\mathrm{x}}$ showing the structural and magnetic transformation temperatures. The concentrations of the samples used are pointed to by arrows. The composition region of Invar and anti-Invar are shown on the top of the figure.

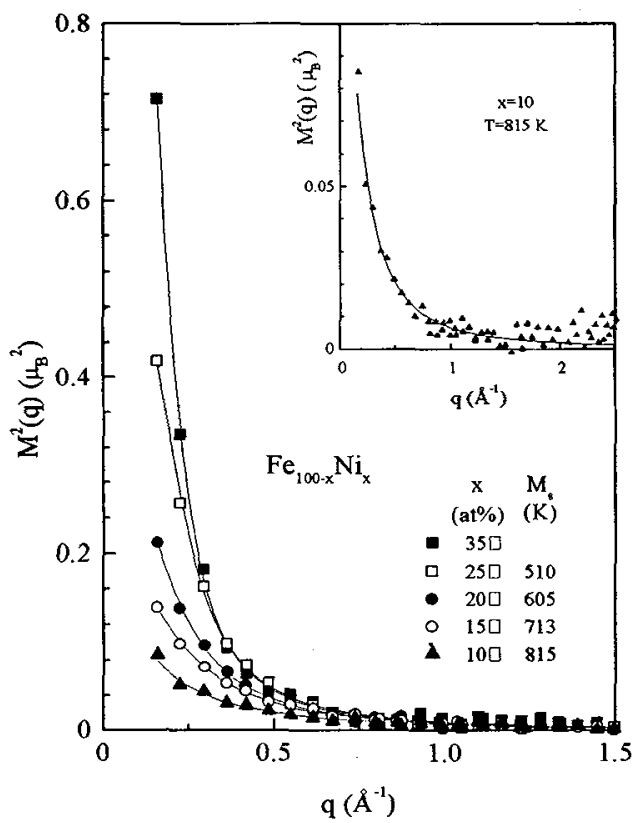

Fig. 3. The magnetic cross section as a function of $\mathrm{q}$. The inset shows the absence of $\mathrm{AF}$ correlations in $\mathrm{x}=10$

temperatures. In recent neutron diffraction studies we have in fact shown for anti-Invar that the fluctuations of the magnetic moment, as well as the associated correlation length, can actually increase with increasing temperature [7].

The relative differences in the free energies of the bcc, fcc and hcp phases of $\mathrm{Fe}$ are shown in figure 1 [810]. Curve 1 is the case in which the bcc phase is $F M$ and stable up to the $A_{3}$ point. Between $A_{3}$ and $A_{4}$ the fcc phase is stable and above $A_{4}$ bcc appears once more. Curve 2 shows that if the $\alpha$ phase is deprived of FM exchange it is no longer stable at all. In this case it remains to compare the free energies of $\epsilon$ and $\gamma$ as in curve 3 , which shows that $\gamma$ would be stable down to about $450 \mathrm{~K}$ below which $\epsilon$ is expected to stabilize. If, on the other hand, the anti-Invar effect is suppressed the $\alpha$ phase with FM coupling becomes stable throughout the temperature range (curve 4). This thermodynamic analysis shows that without the anti-Invar effect fcc Fe would not have been stable. To show this directly we present results on paramagnetic neutron diffraction experiments on $\mathrm{Fe}_{100-\mathrm{x}} \mathrm{Ni}_{\mathrm{x}}$ anti-Invar alloys with $\mathrm{x}=35,30,20,15$ and 10 at $\%$ in their fcc stability ranges and examine the influence of the local magnetic moment on the stability of the fcc phase and compare the results to those obtained from thermodynamical analysis.

\section{EXPERIMENT}

The experiments were carried out on the D7 spectrometer at ILL-Grenoble. This instrument is a multidetector unit capable of performing $x-y-z$ polarization analysis, whereby the magnetic scattering can be separated independent of any particular model. The wavelength of the neutrons was $4.83 \AA$ and measurements were taken up to $q=2.5 \AA^{-1}$. The polycrystalline samples were $15 \mathrm{~mm}$ diameter and $110 \mathrm{~mm}$ long hollow cylinders with $2 \mathrm{~mm}$ thickness. This geometry was chosen to optimize the transmission and to reduce multiple 


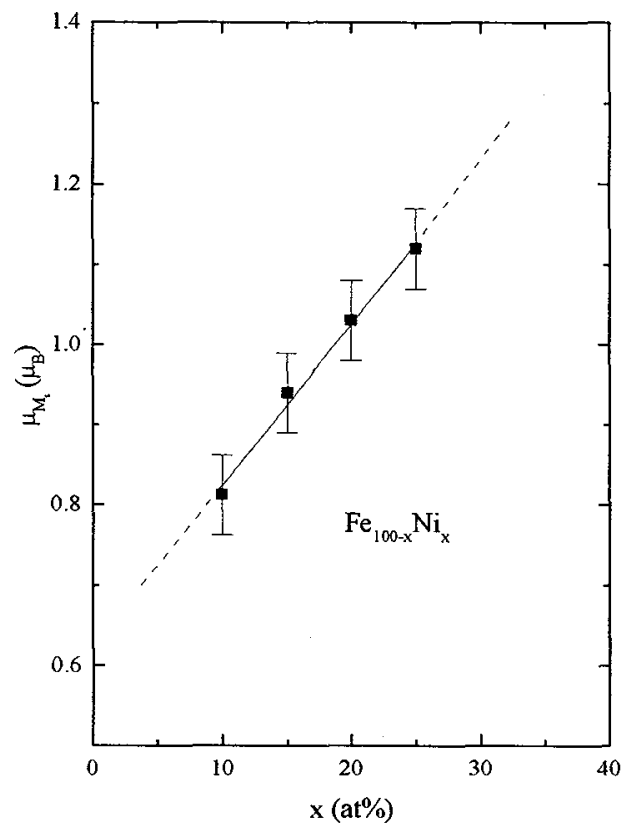

Fig. 4. The concentration dependence of the magnetic moment at $\mathrm{M}_{\mathrm{s}}$. The moment approaches 0.6 for fcc $\mathrm{Fe}$ at the $\mathrm{A}_{3}$ point.

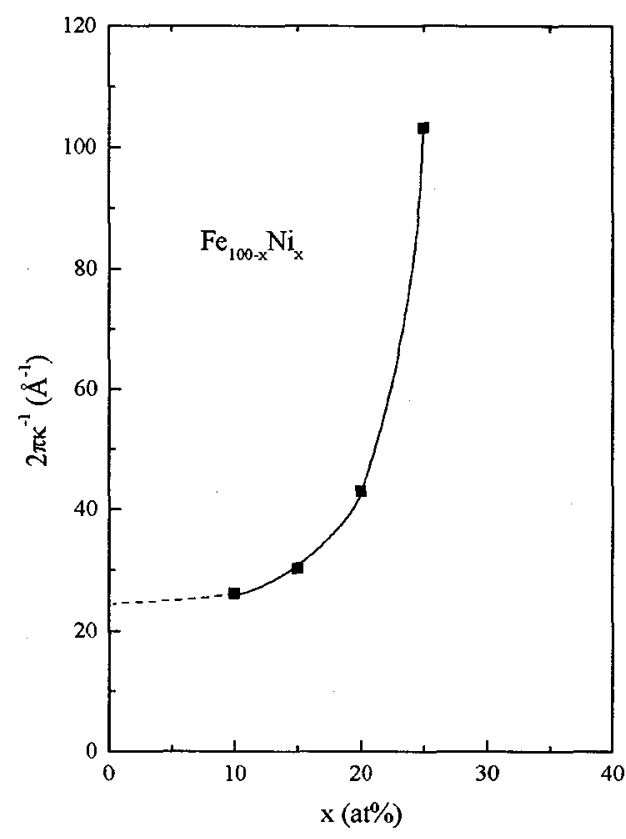

Fig. 5. The concentration dependence of the spatial correlation length at $\mathrm{M}_{\mathrm{S}}$.

scattering. The sample was fitted in an oven assembly which could attain temperatures in access of $1200 \mathrm{~K}$ with a long term temperature stability within $2 \mathrm{~K}$. The neutrons were incident on the mid $50 \mathrm{~mm}$ section of the cylinder. The concentration of the samples, checked by microprobe analysis, do not vary more than $0.5 \%$ of their nominal value throughout the length. The phase diagram of Fe-Ni alloys on the Fe-rich side showing the martensitic and austenitic start (s) and finish (f) temperatures $M_{s}, M_{f}, A_{s}, A_{f}$, are shown in figure 1 where the arrows are drawn to the concentrations of the samples used in the present investigation.

\section{RESULTS}

Prior to the experiments the temperature of the sample was raised through $A_{f}$ and then lowered to near $M_{s}$. The flipping ratio served to monitor $M_{s}$ since, below this temperature the ratio deteriorates when long range FM order sets in along with the martensitic phase and depolarizes the neutrons. The results are shown in figure 2 where the amplitude of the spin fluctuations $\mathrm{M}^{2}(\mathrm{q})$ is plotted as a function of the wave vector $\mathrm{q}$. A strong foreward scattering is observed for all samples indicating FM short range correlations although no long range FM ordering is expected to occur in the samples with $\mathrm{x} \leq 20$ and perhaps only a weak one with a low $\mathrm{T}_{\mathrm{C}}$ for $x=25$. On the contrary $A F$ ordering is expected to take place for $x \leq 20$ as seen in the phase diagram in fig. 1. The inset shows in fig. 2 the absence of $A F$ correlations in the $x=90$ sample at $815 \mathrm{~K}$, which would have appeared as peak like structures at $q=1.5 \AA^{-1}$ and $q=2.5 \AA^{-1}$. The lines drawn through the data are Lorenzian fits from which the correlation length $\lambda_{c}$ is determined from the half width $\kappa$ as $\lambda_{c}=2 \pi \kappa^{-1}$. The local magnetic moment is determined by integrating $\mathrm{M}^{2}(\mathrm{q})$ over all $\mathrm{q}$ up to the boundary of the Brillouin zone as

$$
\mu=\frac{\int_{Z B} M(q)\left(4 \pi^{2} q^{2}\right) d q}{\int_{Z B} 4 \pi^{2} q^{2} d q} .
$$


The magnetic moment at $M_{s}$ and the correlation length likewise at $M_{s}$ as a function of concentration are given in figure 4 and figure 5 respectively. The magnetic moment increases with increasing almost linearly with concentration as the $M_{s}$ temperature decreases. By extrapolating to $x=0$ one finds $\mu \approx 0.6 \mu_{B}$ for fcc-Fe at the $\mathrm{A}_{3}$ point. The correlation length likewise increases with increasing Ni concentration.

\section{DISCUSSTON}

The increase of the local magnetic moment with increasing Ni content is contrary to expectations. Since Ni has a magnetic moment of only about $0.2 \mu_{\mathrm{B}}$ around $1200 \mathrm{~K}$ and fcc Fe about $0.9 \mu_{\mathrm{B}}$ at similar temperatures [11], one would expect the average magnetic moment to decrease with increasing Ni concentration. At the same time one must keep in mind that the presence of FM corrrelations in a system where the ground state is $\mathrm{AF}$ is also an unusual situation in itself.

These discrepancies can be resolved if one considers the anti-Invar nature of fcc Fe and its alloys with Ni. The presence of FM correlations within the stability range of fcc Fe is due to the tendency of the stabilization of the HM state at high temperatures [4]. If the HM state were fully stable its magnetic moment would have reached about $2.8 \mu_{\mathrm{B}}$. In this case the addition of $\mathrm{Ni}$ would have actually reduced the average magnetic moment. However, in the real case the HM state is not fully stabilized due to the strongly fluctuating magnetic moment. Furthermore, recent experiments show that the local magnetic moment can actually increase with increasing temperature [7]. On the other hand the moment of $\mathrm{Ni}$ compared to $\mathrm{fcc} \mathrm{Fe}$ is stable and therefore, the addition of $\mathrm{Ni}$ furthers the stabilization of the $\mathrm{HM}$ state of the alloy. This is observed by the increase of the correlation length as the amount of $\mathrm{Ni}$ increases. As the magnetic moment and correlation length increase $\mathrm{M}_{s}$ decreases and the fcc state is stabilize down to lower temperatures. From this it can be concluded that the stabilization of the HM state leads to the stabilization of the fcc state, which comes in exact agreement with the results of thermodynamical analysis. The local moment in the paramagnetic state of the alloys cannot increase indefinitely with increasing Ni concentration and has to begin to decrease to the value of $\mathrm{Ni}$ above a certain Ni composition. This should happen at around 60 at $\% \mathrm{Ni}$ which is the composition for which $\mathrm{T}_{\mathrm{C}}$ of the alloy series is a maximum.

\section{Acknowledgements}

We acknowledge gratefully U. Brill, P. Fischer and B. Gehrmann of Krupp VDM for the generous support in preparing the samples and $\mathrm{H}$. Bach and P. Stauche for sample preparation and characterization. We also thank J. Kästner for helpful comments and $\mathrm{H}$. Zähres for technical assistance. We are indebpted to W. Pepperhoff for his continued interest and support. This work was supported by the Deutsche Forschungsgemeinschaft (SFB 166) and ILL-Grenoble.

\section{References}

[1] Wassermann E.F., Ferromagnetic Materials (North Holland, Amsterdam, 1990) pp. 237.

[2] Wassermann E.F., J. Magn. Magn. Mater. 100 (1991) 346

[3] Keune W., Ezawa T., Mecedo W.A.A., Glos U., Schletz K.P., and Kirschbaum U., Physica B 161 (1989).

[4] Acet M., Zähres H., Wassermann E.F. and Pepperhoff W., Phys. Rev. B 49 (1994) 6012.

[5] Acet M., Schneider T., Zähres H., Wassermann E.F., and Pepperhoff W., J. Appl. Phys. 75 (1994) 7015.

[6] Schneider T., Acet M., Rellinghaus B., Wassermann E. F. and Pepperhoff W., Phys. Rev. B 151 (1995) 8917-8921.

[7] Acet M., Wassermann E. F., Andersen K., Murani A. and Schärpff O., Phys. Rev. B, to be published.

[8] Stepakoff G. and Kaufmann L., Acta Metall. 16 (1968) 13.

[9] Kaufmann L. and Bernstein H., Computer Calculations of Phase Diagrams, (Academic Press, N.Y., London 1970).

[10] Bendick W. and Pepperhoff W., Acta Metall.30 (1982) 679.

[11] Brown P.J., Capellmann H., Deportes J., Givord D., and Ziebeck K.R.A., J. Magn. Magn. Mater. 30 (1983) 335. 